\title{
Antwort der Autorin „Wunschkinder - Fördernde und hemmende Einflüsse medizinisch assistierter Reproduktion auf die Persönlichkeits- und Beziehungsentwicklung" zum Leserbrief Wischmann et al.
}

\author{
Karin J. Lebersorger
}

Online publiziert: 1. September 2016

(c) Springer-Verlag Wien 2016

\section{Sehr geehrte Herausgeber!}

Ich bedanke mich für die Auseinandersetzung, sie gibt mir die Möglichkeit, dem aus meiner Sicht fundamentalen Missverständnis zu entgegnen, das dem Brief an die Herausgeber zugrunde zu liegen scheint.

Ausgehend von der stetig steigenden Zahl von Kindern nach medizinisch assistierter Reproduktion, von denen immer mehr psychotherapeutische und kinderpsychiatrische Hilfe benötigen, wendet sich der Beitrag nicht gegen die Reproduktionsmedizin und pathologisiert Eltern keineswegs. Vielmehr zeigt er aus einem tiefenpsychologisch-psychodynamischen Blickwinkel bewusste und unbewusste Dynamiken auf, deren Verstehen für die Beratung und Begleitung der Familien hilfreich ist.

So werden die intrapsychischen Prozesse der außerordentlichen psychischen Belastung beider Elternteile beschrieben, auf die die Leitlinie zur „Psychosomatisch orientierten Diagnostik und Therapie bei Fertilitätsstörungen “1 in den den Kap. 2.1.2 und 3.1.1 hinweist.

Die explizite Benennung früher Abwehrformen, die bei großem psychischen Stress punktuell aktiviert werden, um den psychischen Gleichgewichtszustand aufrecht zu erhalten, soll nachvollziehbar machen, warum es für Eltern später so schwer sein kann, für die reproduktionsmedizinische Behandlung Worte zu finden. In diesem Kontext sind Spaltung, Verleugnung und Verdrängung nicht pathologisch. Nur wenn frühe Abwehren durchgängig auftreten, wie etwa bei

${ }^{1}$ http://www.awmf.org/leitlinien/detail/1l/016-003.html [08.05.2016]

Dr. K. J. Lebersorger $(\varangle)$

Institut für Erziehungshilfe - Child Guidance Clinic,

Schlosshoferstraße 4/7/3, 1210 Wien, Österreich

karin.lebersorger@chello.at

Persönlichkeitsstörungen, ist eine Psychopathologie gegeben.

Auch das Aufzeigen narzisstischer Kränkungen stellt keineswegs eine Abwertung dar, sondern beschreibt eine tiefe Verletzung, wie sie entstehen kann, wenn der genuine Wunsch nach einem eigenen Kind auf natürlichem Weg nicht erfüllbar ist.

Werden die elterlichen Belastungen nicht verarbeitet, kann die Entstehungsgeschichte des Kindes zu einem Familiengeheimnis werden, welches sich auf die Eltern-Kind-Beziehungen negativ auswirkt. Daher findet sich die Forderung nach Aufklärung der Kinder über die Art ihrer Konzeption auch im Kap. 3.2.4 der Leitlinie.

Aspekte von Heimlichkeit und unerfüllbaren Wunschbildern betreffen nicht nur Kinder nach Gametenspenden, sondern auch Einlinge von leiblichen Eltern. Werden unerwünschte Gefühle, die die Kinderwunschbehandlung begleiten, nicht integriert, erhalten auch sie großteils keine altersgemäße Aufklärung und Beantwortung ihrer Fragen und finden sich elterlicher Befangenheit gegenüber. Psychologisch-psychotherapeutische Beratung kann für Paare und Eltern diesbezüglich entlastend und hilfreich sein (vgl. Leitlinie 4.3).

Elterliche Belastungen, unerfüllbare Wunschvorstellungen und weitgehende Tabuisierung bilden eine Risikokonstellation, die es durch eine die zugrunde liegende Dynamik verstehende Beratung und Begleitung zum Wohl der Kinder zu minimieren gilt.

Mit freundlichen Grüßen

Dr. Karin J. Lebersorger

Interessenkonflikt K.J. Lebersorger gibt an, dass kein Interessenkonflikt besteht. 